\title{
A New Invention Method to Determine the Reduction Factor for Low Fabric Tension Properties for Head Garment Fabrication
}

\author{
A.F Aiman $^{1, *}$, M.N Salleh ${ }^{2}, K . A$ Ismail $^{1}$ \\ ${ }^{1}$ School of Manufacturing Engineering, Universiti Malaysia Perlis, 02600 Arau, Perlis, Malaysia \\ ${ }^{2}$ School of Technology Management and Logistic, UniversitiUtara Malaysia, 06010 Sintok, Kedah, \\ Malaysia
}

\begin{abstract}
This paper proposes a new method to determine the reduction factor for producing a head garment with specified targeted pressure output. Pressure garment fabric mostly supplied to the local hospitals with no information of the material properties and the fabrication method generally used a single reduction factor at various body segments. Reduction factor defined as the percentage of reducing the garment size from the original circumference of the body part which contributes to the compression. The objective of this study is to compare the fabrication method of head garment using reduction factor equation from previous research with the new proposed method. The equation to predict the reduction factor requires the parameter of the fabric tension which is obtained from tensile test and radius of curvature of the human body parts. In the new proposed method, a 3D scanning was used for data acquisition to obtain the geometry of the head area. The pressure outputs are measured by a pressure measurement system developed from Flexiforce sensor and Arduino circuit board. By using the equation, the result shows the calculated reduction factor produced an extremely tight head garment compared to the conducted experiments which manage to produce an adequate reduction factor with a targeted pressure output of $20 \mathrm{mmHg}$. The result of the experiment indicates that the reduction factor ranging from $17 \%$ to $23 \%$ compared to the equation which produces $20 \%$ to $47 \%$ of reduction factor. As an additional, the proposed experimental method can be used for different type of pressure garment fabrics in order to obtain the relationship between the reduction factor and the circumference of the body parts.
\end{abstract}

\section{Introduction}

Pressure therapy derives from an early surgical principle, in which the use of bandages and splints more than 40 years ago since 1970s [1,2]. Methods of applying pressure have been documented and it included, among others, the method of splints, bandaging, and elastic garments. Pressure garment (PG) mostly used to treat hypertrophic scars because it is an easy device to apply, minimally disruptive to activity, and the availability in terms of

\footnotetext{
* Corresponding author : aiman@unimap.edu.my
} 
commercially or through skilled technician. Regarding the skilled technician, mostly in medical or hospital departments they are known as an Occupational Therapist (OT). Besides that, PG manage to correct the deformity and eventually decrease the need for corrective surgery.

Even though there are still debates among researchers about the ideal pressure exerted by the pressure garment, most researchers agreed that the best pressure exerted from the compression of pressure garment is ideally in the range of $15 \mathrm{mmHg}$ to $24 \mathrm{mmHg}$ or 1999.85Pato 3199.74Pa [1, 3, 4]. It has been reported that pressure greater than $40 \mathrm{mmHg}$ may result other complications [5]. Nevertheless, most pressure garments fitting were assessed using "sight, touch and experience" $[2,6]$. In order to predict the pressure generated by pressure garment, Laplace's Law has been widely used by other previous studies as given in Eq. 1 [7-12]. The parameters involved in the equation are the fabric tension and the radius of curvature. Both parameters can be obtained through tensile test and the dimension of the circumference of human body parts.

$$
\text { Pressure }, P(P a)=\frac{\text { Tension in the cylinder wall }, T\left(\mathrm{Nm}^{-1}\right)}{\text { Radius of the cylinder }, r(\mathrm{~m})}
$$

\section{Problem statements}

The Laplace`s Law was well known used in predicting the pressure output from the usage of the pressure garment. The important parameters known are the tension of the fabric and the circumference of the body parts. The tension of the fabric is determined from the standard tensile test and circumference is measured from the human body parts. Regarding the fabric used for this study, five specimens were tested by tensile test using MS ISO 13934-1:2003 standard. The modulus of elasticity calculated from the result of the tensile test was $246 \mathrm{~N} / \mathrm{m}$.

In order to obtain the circumference of head area, a 3D scanner was used to capture the geometrical of a mannequin head. The head segment divide to 20 layers of section-cut given by $10 \mathrm{~mm}$ spacing in horizontal direction as shown in Fig. 1.
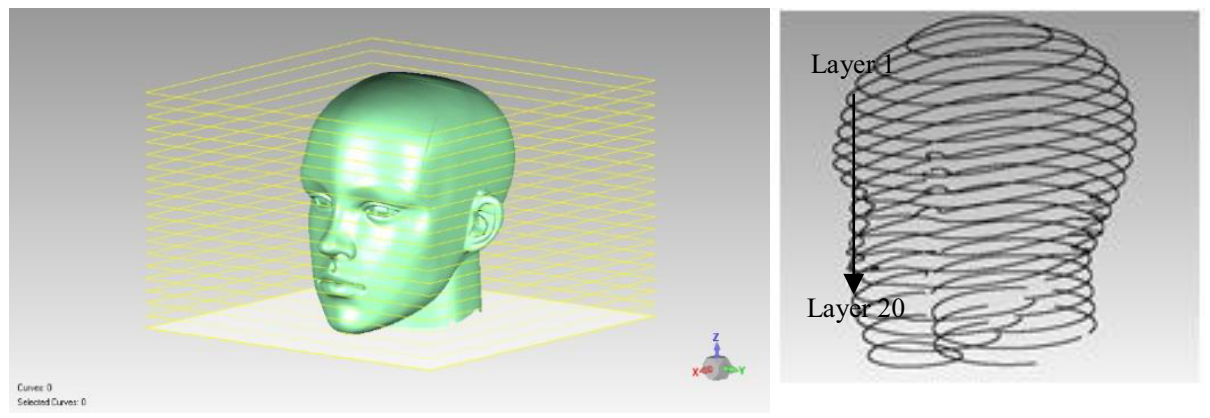

Fig. 1. 3D scanning for head segment using the mannequin`s head.

As for the reduction factor, which refers to the percentage of the reduced dimension from the original circumferences in order to deliver the exerted pressure, the prediction calculated by using Eq. $2[13,14]$.

$$
R e=\frac{1}{1+\frac{E}{P r}}
$$


Based on the Eq. 2, there are three main parameters needed to calculate the reduction factor $(\mathrm{Re})$, fabric tension in $\mathrm{N} / \mathrm{m}(\mathrm{E})$, the pressure to be exerted $(\mathrm{P})$ in Pascal and the radius of curvature (r) in meter. Using Equation (2), the reduction factor calculated value ranging from $20 \%$ to $47 \%$ of each section. The results indicate that the reduction factor produced was extremely big and resulted a tight head garment to be fitted to the mannequin's head. Reduction factor calculated using the fabric tension from the tensile test cannot be applied for the larger circumferences. According to previous studies, the reduction factor used for the most body part are in the range from $10 \%$ to $25 \%$ only $[6,15,16]$. Therefore, a new method is proposed to determine the relationship of reduction factor and circumference to achieve certain target pressure output.

\section{Method}

\subsection{Experiment using a cylinder to determine the reduction factor for head segment}

An experiment using several different sizes of round Polyvinyl Chloride (PVC) cylinder pipes with different circumference dimension range from $28 \mathrm{~cm}$ to $64 \mathrm{~cm}$ designed to investigate the reduction factor use to achieve a certain pressure output. The outer diameter of the cylinder was selected based on the range of the circumference size of a human head. In this study, the pressure output was set to $20 \mathrm{mmHg}$ or $2666.4 \mathrm{~Pa}$. A pressure sensor measurement system was developed from Flexiforce sensor and Arduino UNO board to measure the pressure output. The sensor is placed under the fabric and then monitored to achieve the targeted pressure output and the length which refer to the reduction factor is marked.

Three samples were used for each cylinder and the average of the reduced length were taken to make a validation sample. The validation sample is stitched and measured to validate either the pressure obtain as targeted. For each cylinder, fabric with $10 \mathrm{~mm}$ in width used for the experiment. The length of each cylinder depends on the circumference size of each cylinder. Each sample is marked with the original circumference and labelled as original circumference. A scale with $5 \mathrm{~mm}$ distance was drawn before the fabric being stretched as shown in Fig. 2 (a).

A jig was designed to replicate the stitch area located at the PVC area and the specimen will be inserted through the hole and wrap through the PVC and went through again in the same hole as the intake as shown in Fig. 2 (b). This procedure is done carefully to make sure the alignment according to the PVC circumference. A stopper was made at the intake of the fabric to ensure the fabric is locked in position when the fabric being pulled from the other direction. While the fabric being pulled and the Flexiforce sensor measure up to the targeted pressure, the measurement was being monitored continuously. Once the reading achieved the targeted pressure, the specimen locked using a clamp and the reading was monitored until the pressure targeted reading stabilized and marked the reduction length on the scale made previously. The length for the reduction factor recorded as shown in Fig. 2 (c). The procedures are repeated to all of the fabric specimens.

In order to validate the results, a validation sample using the mean value of the reduced length of the three samples. The mean value of the reduced length will be fabricated by stitching the fabric and measured to validate the pressure output achieved the specified targeted pressure output which is $20 \mathrm{mmhg}$ as shown in Fig. 2 (d). 


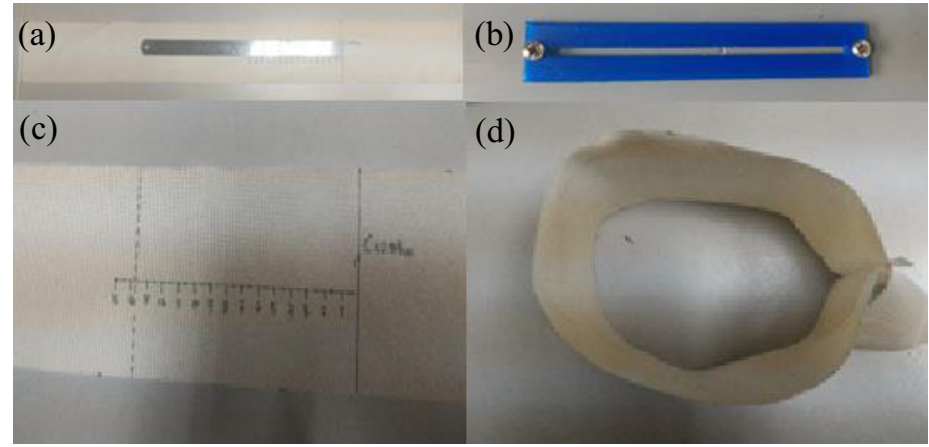

Fig. 2.(a) Sample of specimen (b) Jig for specimen (c) Length measured for the reduction factor (d) Sample from mean data stitched to verify with the pressure output.

\subsection{Pressure measurement system (PMS)}

Pressure measurement system was developed using Flexiforce sensor from Tekscan, USA which have been used in previous studies[17-19]. The pressure measurement used 1lb low force sensor. Arduino board was used as a combination to flexi force sensor for data acquisition. A $10 \mathrm{~g}$ to $50 \mathrm{~g}$ standard weight used to determine the linearity of the sensor output. The amount of the weight was selected due to the maximum pressure output calculated based on the formula of pressure equal to the force over area. The weight in Newton $(0.49 \mathrm{~N})$ divides by the sensor active area, 0.000713 in square meter produced pressure of $6877.03 \mathrm{~Pa}$ or $51.58 \mathrm{mmHg}$. The output from the pressure measurement system converted to $\mathrm{mmHg}$ units because of the units are generally used in rehabilitation department. Fig. 3 (a), (b), and (c) show the diagram, sensor, and experiment setup.

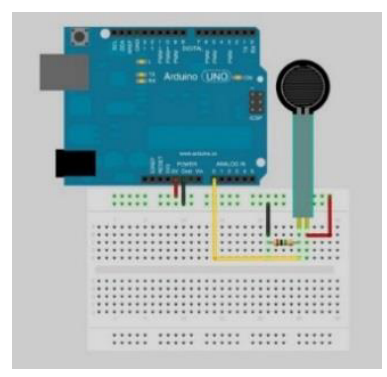

(a)

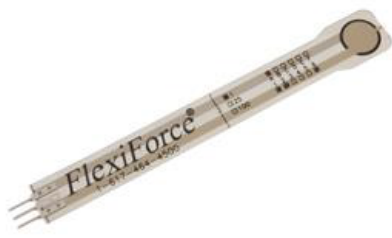

(b)

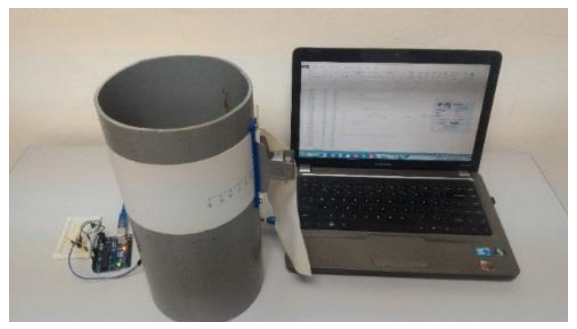

(c)

Fig. 3. (a) Fritzing wiring diagram (b) Flexiforce A201 1lb pressure sensor (c)Experiment setup. 


\section{Result and discussion}

Table 1 shows the results of the length for the reduction gained by each specimen. A graph was plotted and polynomial curve fit was used to determine the equation that will be used to predict the reduction factor for certain circumferences. Fig. 4 shows the graph of the reduction factor that should be used to achieve the specified pressure output. As for the validation sample, the results of the pressure output show the reading of $20 \mathrm{mmhg}$ with tolerance of $+/-1$ mmhg.

Table 1. Reduction factor for achieved specified pressure output.

\begin{tabular}{|c|c|c|c|c|c|c|c|}
\hline & \multicolumn{3}{|c|}{ Length reduction(cm) to achieve } & & & & \\
\hline $\begin{array}{c}\text { 20mmhg } \\
\text { Cylinder } \\
\begin{array}{c}\text { Circumference } \\
(\mathbf{c m})\end{array}\end{array}$ & $\begin{array}{c}\text { Specimen } \\
\mathbf{1}\end{array}$ & $\begin{array}{c}\text { Specimen } \\
\mathbf{2}\end{array}$ & $\begin{array}{c}\text { Specimen } \\
\mathbf{3}\end{array}$ & SD & Mean & cv & $\begin{array}{c}\text { Reduction } \\
\text { factor (\%) }\end{array}$ \\
\hline 27.91 & 3.50 & 3.60 & 3.20 & 0.21 & 3.43 & 6.10 & 12.30 \\
\hline 35.89 & 7.40 & 7.20 & 7.30 & 0.10 & 7.30 & 1.40 & 20.34 \\
\hline 43.87 & 10.60 & 10.70 & 10.10 & 0.32 & 10.5 & 3.10 & 23.86 \\
\hline 52.84 & 13.70 & 13.50 & 13.60 & 0.10 & 13.6 & 0.70 & 25.74 \\
\hline 63.80 & 17.10 & 16.80 & 17.20 & 0.21 & 17.0 & 1.20 & 26.70 \\
\hline
\end{tabular}

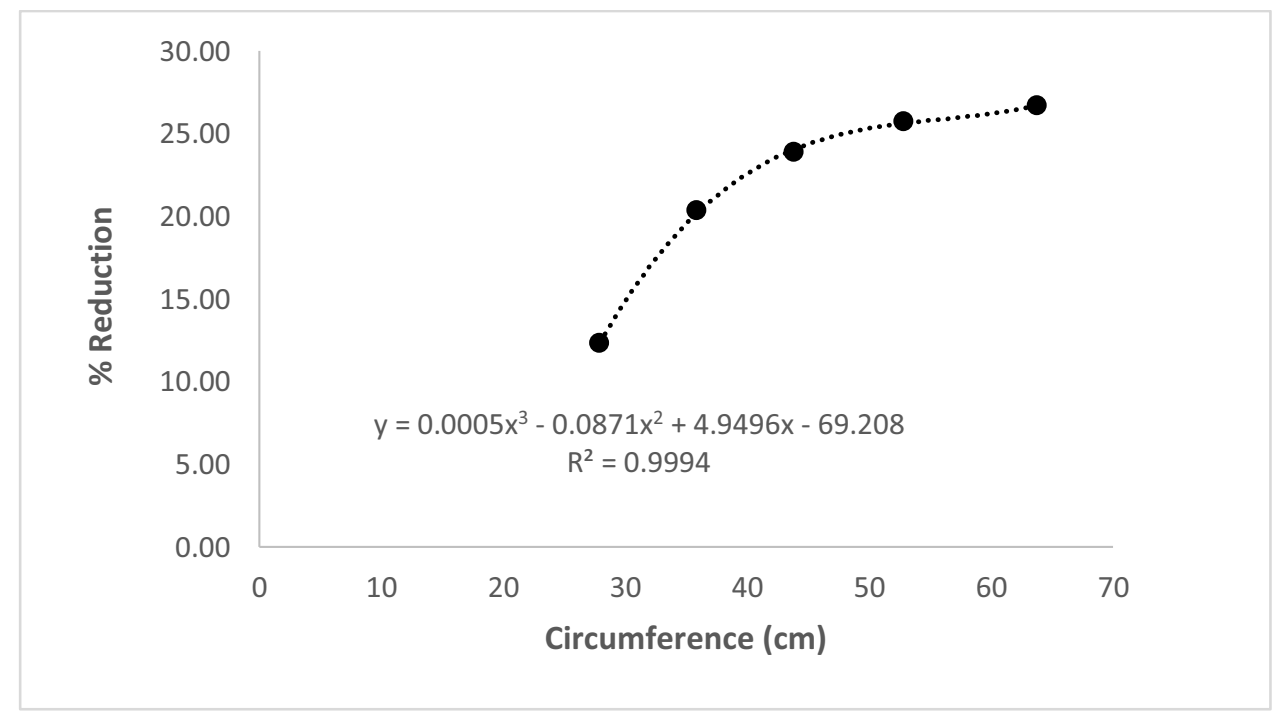

Fig. 4. A 3rd order Polynomial equation of the reduction factor for achieved $20 \mathrm{mmHg}$ pressure output.

Then, using the equation derived from the curve fitting using a 3rd order polynomial equation, the reduction factor is calculated for each section from the scanning data and compared with the prediction of the reduction factor calculated from the Eq. 2 as shown in Table 2. Comparison of the reduction factor is depicted in Fig. 5. 
Table 2. Comparison of reduction from tensile method and variable cylinder circumference method

\begin{tabular}{|c|c|c|c|}
\hline $\begin{array}{c}\text { Section } \\
\text { layer }\end{array}$ & $\begin{array}{c}\text { Original } \\
\text { Circumference(cm) }\end{array}$ & $\begin{array}{c}\text { Reduction factor calculated } \\
\text { from Eq.(2) }\end{array}$ & $\begin{array}{c}\text { Reduction factor from } \\
\text { experiment }\end{array}$ \\
\hline 1 & 14.74 & 0.20 & -0.14 \\
\hline 2 & 33.23 & 0.36 & 0.17 \\
\hline 3 & 41.61 & 0.42 & 0.22 \\
\hline 4 & 46.39 & 0.44 & 0.23 \\
\hline 5 & 49.50 & 0.46 & 0.23 \\
\hline 6 & 50.91 & 0.47 & 0.23 \\
\hline 7 & 51.81 & 0.47 & 0.23 \\
\hline 8 & 52.15 & 0.47 & 0.23 \\
\hline 9 & 51.11 & 0.47 & 0.23 \\
\hline 10 & 48.32 & 0.45 & 0.23 \\
\hline 11 & 47.27 & 0.45 & 0.23 \\
\hline 12 & 46.52 & 0.45 & 0.23 \\
\hline 13 & 45.29 & 0.44 & 0.23 \\
\hline 14 & 44.09 & 0.43 & 0.23 \\
\hline 15 & 42.26 & 0.42 & 0.22 \\
\hline 16 & 42.38 & 0.42 & 0.22 \\
\hline 17 & 41.88 & 0.42 & 0.22 \\
\hline 18 & 40.92 & 0.41 & 0.22 \\
\hline 19 & 40.24 & 0.41 & 0.22 \\
\hline 20 & 39.65 & 0.41 & 0.21 \\
\hline
\end{tabular}
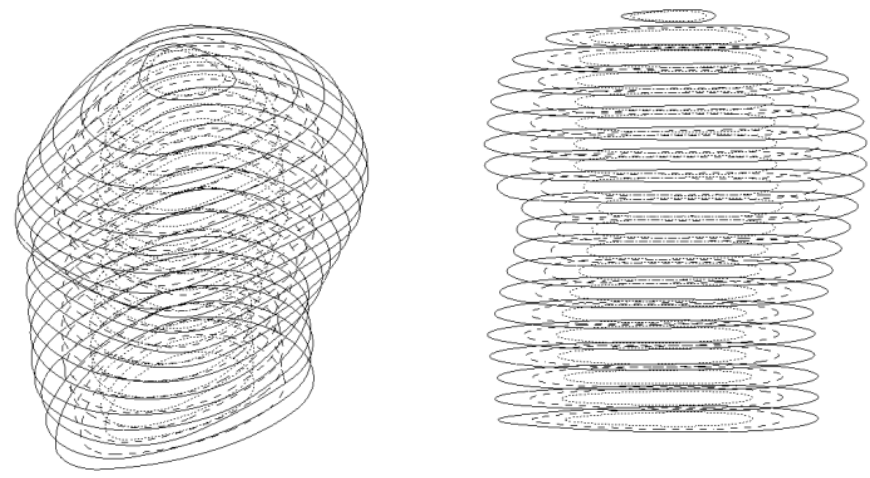

Original circumferences

Re from experiment

Re Calculated from Equation (2)

Fig. 5. Comparison of the Reduction Factor (Re) between Equation (2) and experiment. 
In order to verify the polynomial equation, a different cylinder circumference from the experiment was selected. A cylinder with a $37.5 \mathrm{~cm}$ circumference was selected and the reduction factor calculated was $20.28 \%$. A sample was fabricated and based on this reduction factor, the pressure generated was measured and the result showed the pressure output at $20 \mathrm{mmHg}$ with fluctuations of $1 \mathrm{mmhg}$ to $2 \mathrm{mmHg}$ due to the drift of the sensor shown in Fig. 6.

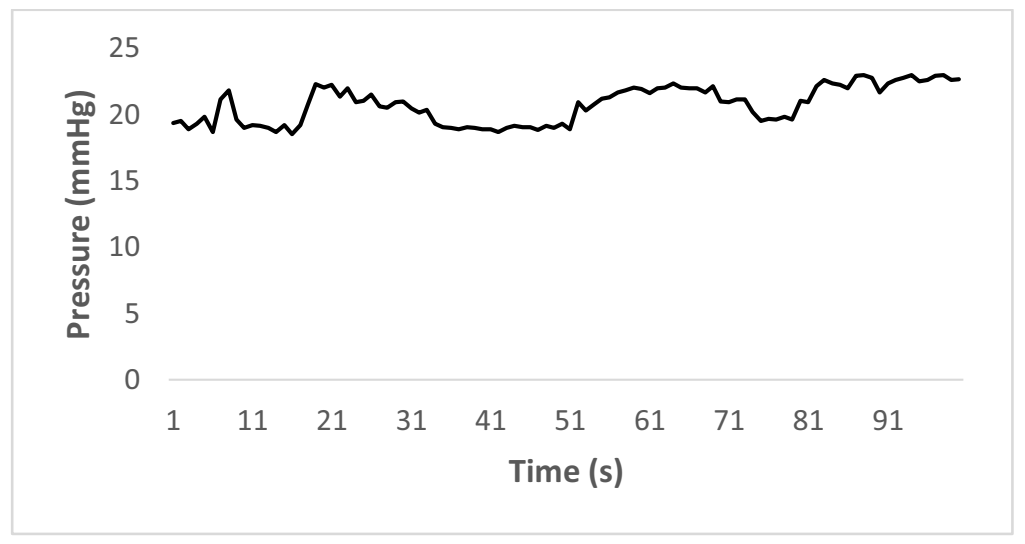

Fig. 6. Validating the polynomial curve equation using cylinder with a $37.5 \mathrm{~cm}$ circumference.

Based on Fig.5 and Table 2, the cylinder experiment indicates the reduction factor is in the range of $17-23 \%$, while the range is $20-47 \%$ by using the Eq. 2 . However, the cylinder experiment shows that at small circumference, at layer 1 , indicating a negative value which refers that the reduction factor is too large to apply at the small circumference. Therefore, no reduction factor should be applied to the small circumference below $30 \mathrm{~cm}$. This result supported the finding from previous research that reported the circumference below $30 \mathrm{~cm}$ does not provide an accurate results by using prediction calculated from the Laplace Law [4]. Even though the reduction factor indicates $20 \%$ of the reduction factor at layer 1 , the result of pressure measurement using sensor indicates a high value exceeding $20 \mathrm{mmHg}$. However, in this study, the tension of the fabric is slightly low, value of $246 \mathrm{~N} / \mathrm{m}$ which resulted a bigger reduction factor compare to the previous research that used a fabric with higher value of fabric tension which is more than $400 \mathrm{~N} / \mathrm{m}[7,20]$. Therefore, the experiment using cylinders can be used as an alternative method to calculate the reduction factor for a low fabric tension parameter. Based on the experiment results, the reduction factor from the cylinder experiment indicate an acceptable reduction factor to fabricate the head garment.

\section{Conclusion}

The experiments using cylinder provide a result that indicates the reduction factor should be used in producing head garment is about $17 \%$ to $23 \%$. It is shown that the result can be used to produce a head garment that will fit in the mannequin's head compared to the reduction factor calculated from the equation which is up to $47 \%$ of reduction factor that produce a very tight head garment which does not fit for the head. By using the cylinders, the equation from the polynomial graph can be used to predict the reduction factor for different circumferences. As an additional, any type of pressure garment's fabric that's being received by local hospitals with no information about the material properties should 
be tested by this method in order to determine the reduction factor with a targeted pressure output.

We thank the Occupational Therapist staff from the rehabilitation unit of Hospital Tuanku Fauziah, Perlis for providing professional consultation and producing the head garments. Thanks to the Ministry of Higher Education Malaysia for supporting this research under Prototype Research Grant Scheme (PRGS).The authors would like to thank School of Manufacturing, Universiti Malaysia Perlis for providing laboratory facilities for $3 \mathrm{D}$ scanning.

\section{References}

1. L. Macintyre, M. Baird, Burns, 32,10 (2006)

2. P. Joanne, W. Gill, Pressure Garments: A manual on their design and fabrication, (Butterworth-Heinemann, Oxord, 1995)

3. L.H.Y. Candy, L.T.W.P. Cecilia, Z.Y. Ping, Burns, 36,1234(2010)

4. L. Macintyre, Burns, 33, 579 (2007)

5. L.H. Engrav, D.M. Heimbach, F.P. Rivara, M.L. Moore, J. Wang, G.J. Carrougher, B. Costa, S. Numhom, J. Calderon, N.S. Gibran, Burns, 36, 975 (2010)

6. L. Macintyre, M. Baird, Burns, 31,11 (2005)

7. W.Y. Leung, D.W. Yuen, S.P. Ng, S.Q. Shi, J. Burn Care Res., 31, 716 (2010)

8. T. O., E. Ashayeri, B. M., S. A., F. Alam, S. Marteau, Procedia Eng., 2, 2823 (2010)

9. B.M.E. Valentinuzzi, A. J. Kohen, IEEE Pulse, 3, 66 (2012)

10. K.D. Nguyen, A.A. Simard. Meilleur, C. Berthiaume, R. Godbout, L. Mottron, S.F.F. Ng, C.L.P. Hui, N. Yildiz, L. Wang, M. Felder, J.Y. Cai, Burns, 33,59 (2007)

11. N. Yildiz, Burns, 33,59 (2007)

12. L. Macintyre, R. Ferguson, Burns, 39, 1073 (2013)

13. M.N. Salleh, M. Acar, N.D. Burns, 2011 4th International Conference on. IEEE (2011)

14. M.N. Salleh, H.M. Lazim, S.N. Othman, A.F. Merican, International Conference on Advacanced Mechantronic Systems (2012)

15. S.F. Ng, C.L. Hui, Int J Eng Res Appl. 71, 275 (2001)

16. L.T. Nguyen, N.J. Massman, B.J. Franzen, D.H. Ahrenholz, N.W. Sorensen, W.J. Mohr, L.D. Solem, J. Burn. Care. Res., 25, 485 (2004)

17. M. Ferguson. Pell, S. Hagisawa, D. Bain, Med. Eng. Phys., 22, 657 (2000)

18. M. Burke, B. Murphy, D. Geraghty, IEEE SENSORS 2014 Proceedings (2014)

19. J. Li, H. Liu, Y. Wang, L. Shi, F. He, Measurement, 45, 2114 (2012)

20. S.F.F. Ng, C.L.P. Hui, Int. J. Cloth. Sci. Technol., 11, 251 (1999) 